\title{
Age-of-onset-dependent influence of NOD2 gene variants on disease behaviour and treatment in Crohn's disease
}

\author{
Carsten Posovszky ${ }^{1 *}$, Veronika Pfalzer ${ }^{1}$, Georgia Lahr ${ }^{1}$, Jan Hendrik Niess ${ }^{2}$, Jochen Klaus ${ }^{2}$, Benjamin Mayer ${ }^{4}$,
} Klaus-Michael Debatin ${ }^{1}$ and Georg BT von Boyen ${ }^{3}$

\begin{abstract}
Background: Influence of genetic variants in the NOD2 gene may play a more important role in disease activity, behaviour and treatment of pediatric- than adult-onset Crohn's disease (CD).

Methods: 85 pediatric- and 117 adult-onset CD patients were tested for the three main NOD2 CD-associated variants (p.R702W, p.G908R and p.10007fs) and clinical data of at least two years of follow-up were compared regarding disease behaviour and activity, response to therapy and bone mineral density (BMD).

Results: Chronic active and moderate to severe course of $C D$ is associated in patients with pediatric-onset $(p=0.0001)$ and NOD2 variant alleles $(p=0.0001)$. In pediatric-onset CD the average PCDAl-Score was significantly higher in patients carrying NOD2 variants $(p=0.0008)$. In addition, underweight during course of the disease $(p=0.012)$ was associated with NOD2 variants. Interestingly, osteoporosis was found more frequently in patients carrying NOD2 variant alleles ( $p=0.033$ ), especially in pediatric-onset CD patients with homozygous NOD2 variants $(p=0.037)$. Accordingly, low BMD in pediatric-onset $C D$ is associated with a higher PCDAI $(p=0.0092)$, chronic active disease $(p=0.0148)$, underweight at diagnosis $(p=0.0271)$ and during follow-up $(p=0.0109)$. Furthermore, pediatric-onset $C D$ patients with NOD2 variants are more frequently steroid-dependent or refractory $(p=0.048)$ and need long-term immunosuppressive therapy ( $\mathrm{p}=0.0213)$.

Conclusions: These data suggests that the presence of any of the main NOD2 variants in CD is associated with osteoporosis and an age of onset dependent influence towards underweight, higher disease activity and a more intensive immunosuppressive therapy. This observation supports the idea for an early intensive treatment strategy in children and adolescent CD patients with NOD2 gene variants.
\end{abstract}

Keywords: Crohn's disease, NOD2, CARD 15, Osteoporosis, Pediatric-onset

\section{Background}

Inflammatory bowel disease (IBD) is characterized by chronic inflammation of the gastrointestinal tract and can occur at any age. Crohn's disease (CD), ulcerative colitis (UC) and indeterminated colitis are defined according to the clinical, endoscopic, radiological and histopathological features. IBD is a multifactorial disease caused by genetic, bacterial, nutritional, and immunological factors. There is clear evidence for a strong genetic

\footnotetext{
* Correspondence: carsten.posovszky@uniklinik-ulm.de

1 Department of Pediatrics and Adolescent Medicine, University Medical Center Ulm, Eythstr. 24, Ulm 89075, Germany

Full list of author information is available at the end of the article
}

influence in the pathogenesis of IBD, especially in CD, coming from twin studies [1] with disease concordance up to $50 \%$ for monozygotic CD twins. In addition, pediatric-onset $\mathrm{CD}$ is characterized by distinct phenotypic differences compared to adult-onset suggesting that a genetic susceptibility may play an important role [2]. Polymorphisms in the leucine-rich repeats (LRR) region of the NOD2/CARD15 gene were identified as independent risk factors for $\mathrm{CD}$ in Caucasians [3,4], which varies depending on the number of mutations from a 2 to 4-fold increased risk with 1 of the 3 major alleles to 40-fold increase in homozygous or compound heterozygous carriers [5]. The three major polymorphisms in the coding 
region of NOD2/CARD15, associated with $\mathrm{CD}$, are p. R702W, p.G908R, and p.L1007fs. The NOD2 gene is involved in the innate immune response by recognizing muramyl dipeptide of intracellular bacterial lipopolysaccharides and activating nuclear factor $\mathrm{kB}$ pathways [6]. NOD2 is mainly expressed in Paneth cells in the small bowel and is involved in the innate immune response to bacterial pathogens [7]. Thus variant NOD2 alleles are associated with reduced defensin expression in response to bacteria [8]. Children have shorter environmental exposure and therefore genetic factors such as NOD2 variants may have a greater influence in the etiology and severity of CD [9]. Disease behaviour may change over time, thus long-term follow-up data on pediatric-onset disease are needed [10]. Indeed, the few studies analyzing genotype-phenotype in pediatric-onset $\mathrm{CD}$ with longer observation time revealed associations of NOD2 variants with disease severity and risk for surgery $[11,12]$.

Children and adolescents suffering from IBD are at risk of developing osteoporosis. The prevalence of severe demineralization in children and in adults ranges from $4.35 \%$ to $42 \%$ [13]. The pathogenesis of osteoporosis in $\mathrm{CD}$ is not completely understood. Bone mineral density (BMD) is influenced by several factors including chronic or recurrent corticosteroid use, malnutrition or malabsorption, low body mass index (BMI), hypogonadism, immobilisation, vitamin D deficiency [14]. Together these factors result in low turnover bone loss. However, also inflammation may significantly contribute to a high-turnover mineral bone loss in CD [14-16]. Osteoclastogenesis and bone resorption is driven by inflammatory mediators such as tumor necrosis factor (TNF) $\alpha$, Interleukin (IL)-1 $\beta$ and Il-6 in synergy with receptor activator of nuclear factor kappa $B$ ligand (RANKL). RANKL is released by activated T-cells and binds RANK on osteoclastic precursors and thereby induce and activate osteoclasts [17]. Thus a chronic inflammatory state, such as the gut in CD patients, in addition affects bone remodelling and contributes to high turn-over mineral bone loss. The influence of NOD2 genotype on osteoporosis in $\mathrm{CD}$ has not been studied yet.

We therefore wished to investigate the potential relationship between the three major NOD2/CARD15 variants in a well characterized, long term follow up dataset of Crohn patients with pediatric- and adult-onset and inflammatory phenotype, response to therapy and lower bone mineral density.

\section{Methods}

\section{Study population and disease phenotype}

The single center study, approved by the Ethics committee Board of the Ulm University, adheres to the ethical principles of the Helsinki Declaration. 202 patients were recruited in the department of pediatrics and adolescent medicine as well as in the department of internal medicine at the Medical University Center Ulm. The patients or their parents gave written informed consent to participate in the study.

The patient's diagnosis based on clinical, radiological, endoscopic and histopathologic findings, and presence of disease for at least 2 years from diagnosis with follow-up. Patients with uncertain diagnosis or unclassified colonic inflammatory bowel disease were excluded. The pediatriconset cohort included patients with age of onset until the age of 18 and final diagnosis until the age of 19. Parameters including age of diagnosis and duration of disease, sex, disease localization, disease behaviour, family history of IBD, body weight and length, body mass index (BMI), extraintestinal involvement, bone mineral density, medical and surgical therapy and disease activity including hospitalization were updated, analyzed and classified according to the Montreal classification. Disease location and behaviour was defined as involvement at any time during disease. Underweight was defined as BMI below $10^{\text {th }}$ percentile for age. Growth failure was defined as inappropriate growth velocity for age and short stature was defined as height below the $3^{\text {rd }}$ percentile for age on a growth chart. Disease activity was calculated based on PCDAI for children with Crohn's disease [18] or CDAI for adults during regular or emergency visits and given as mean value. An average PCDAI/CDAI score above $\geq 10$ / 150 was defined as chronic active and $\geq 30 / 220$ as moderate to severe disease. In children, a hospitalization of more than two weeks per year due to $\mathrm{CD}$ was given to describe disease activity and the percentage of severe ill patients.

\section{Bone mineral density measurement}

Bone mineral density of the lumbar spine (L2-L4) was evaluated using dual-energy X-ray absorptiometry (DEXA) in patients with inflammatory bowel disease using a Hologic QDR 1000 and expressed as grams per square centimetre. Osteoporosis was defined in children as less than two standard deviations (SD) of BMD adjusted to an age matched population (Z-score) [19] and in adults as BMD compared to a young normal reference mean below 2.5 SD (T-score) [20].

\section{Therapy modalities}

The treatment was according to the German guidelines [21] and blinded to the genotype data. Patients received prednisolon (2 mg/kg up to $100 \mathrm{mg} / \mathrm{d}$ ), budenoside (9 mg/ d), azathioprine (AZA) (2.0-2.5 mg/kg/d), 6-mercaptopurin (6-MP) (1-1.5 mg/kg/d), methotrexate (MTX) $\left(15 \mathrm{mg} / \mathrm{m}^{2} /\right.$ week s.c.), infliximab, a chimeric monoclonal antibody to TNF $\alpha$ ( $5 \mathrm{mg} / \mathrm{kg}$ at weeks $0,2,6$ and every 8 weeks), or adalimumab, a humanized monoclonal antibody to TNF $\alpha$ 
(80 mg starting dose, followed by $40 \mathrm{mg}$ every 8 weeks in adults or dose-adjusted for children $24 \mathrm{mg} / \mathrm{m}^{2}$ and adapted for through level). Patients with chronic active disease who did not respond to steroid therapy or relapsed within the first 4 weeks were defined as steroid-dependent or steroid-refractory respectively. These patients were then switched to an immunosuppressive long-term therapy with AZA, 6-MP, MTX or TNF- $\alpha$ antagonists. Patients with pediatric-onset received TNF- $\alpha$ antagonists as second line therapy if enteral therapy, AZA, 6-MP or MTX failed to induce stable remission, withdrawal of steroid treatment was impossible and surgical treatment was not reasonable or not accepted by the patients or their guardians.

\section{DNA extraction and genotyping of NOD2 variants}

Genotyping of the three main NOD2 variants p.R702W (rs2066844, exon 4), p.G908R (rs2066847, exon 8), and p.1007fs (rs20066847, exon 11) were performed in 85 pediatric and 117 adult CD patients of Caucasian origin. Therefore peripheral blood mononuclear cells (PBMCs) of the patients were obtained. Genomic deoxyribonucleic acid (DNA) was extracted from PBMCs using standard methods using QIAmp Flexi Gen DNA Kit (Qiagen, Hilden, Germany). Exons 4, 8 and 11 of the NOD2 gene were individually amplified by polymerase chain reaction (PCR) analysis using standard methods. PCR-samples were sequenced using the CEQ Quick Start Kit on a GeXP Genetic Analysis System (Beckman Coulter, Krefeld) and compared with wild-type NOD2-gene sequence.

\section{Statistical analysis}

Absolute and relative frequencies were used to describe qualitative variables descriptively, and mean with standard deviation as well as median with interquartile range for continuous variables. Differences between frequencies of qualitative characteristics in CD patients carrying NOD2 variants or wild-type were compared using chi-square test or Fishers exact test, respectively. Mann-Whitney or Kruskal-Wallis tests were applied to compare medians and T-test to compare means, respectively. A $p$ value $\leq 0.05$ was considered to be significant. Descriptive statistics for quantitative values were given as median and interquartile range in box plot diagrams using Graphpad Prism software. All statistical tests are interpreted explorative. To adjust for possible confounding, multivariate regression analyses have been conducted in the course of a sensitivity analysis.

\section{Results}

Demographic and disease characteristics of the study population

Data regarding NOD2 genotype, age of onset, average disease duration, disease location and behaviour were obtained from two hundred and two German patients with adequate follow-up and are presented in Table 1 according to the age of onset of CD. NOD2 variant alleles were found in 37 out of 85 pediatric- (44\%) and 47 out of 117 adult- (40\%) onset CD patients (Table 1). No significant differences were noted in the distribution of the three main NOD2 allele variants (data not shown), however the presence of two variant alleles trend to be higher in pediatric-onset than in adult-onset patients $(\mathrm{p}=0.12)$ (Table 1). The mean follow-up was around 11 years in the pediatric-onset group and 16 years in the adult-onset group. Interestingly, the prevalence of IBD in first- or second-degree relatives of $\mathrm{CD}$ patients is statistically more frequent in adult-onset CD patients with NOD2 variants in contrast to NOD2 wild-type (29\% versus $6 \%$; $=0.0023$ ) but not in the pediatric-onset $C D$ patients (Figure 1).

Carrying at least one NOD2 variant allele is associated with an ileocolonic (L3) localization $(p=0.048)$ in the pediatric- and a trend to an ileal involvement alone in the adult-onset $\mathrm{CD}$ patients (38\% versus $21 \%$; $\mathrm{p}=0.06$ ), while pediatric-onset NOD2 wild-type patients more frequently had a colonic involvement alone $(\mathrm{p}=0.048)$. The influence of NOD2 variants towards complicated disease behaviour (B2 or B3) is more pronounced in patients with pediatric-onset $C D(p=0.026)$. Stricturing disease behaviour (B2) is significantly associated with NOD2 variant alleles in pediatric-onset $(\mathrm{p}=0.005)$ and adult-onset CD Patients $(\mathrm{p}=0.051)$ (Table 1).

\section{Pediatric-onset CD patients carrying NOD2 variant alleles} display a higher disease activity and underweight

There is no significant influence of the main NOD2 variants in patients with $C D$ towards extraintestinal manifestation, need for surgery or complications (Figure 1). However, we found chronic active or high active course of disease in CD patients associated with pediatric-onset of disease $(\mathrm{p}=0.0001)$ and any of the main NOD2 variant alleles $(\mathrm{p}=0.0001)$. The average PCDAI-Score over time was significantly higher in pediatric-onset CD patients with at least one NOD2 variant than two wild-type alleles (23.2 versus 14.1; $\mathrm{p}=0.0008$ ) (Figure 2A), and a chronic active (PCDAI $\geq 10$ or $\mathrm{CDAI} \geq 150 ; \mathrm{p}=0.017$ ) or moderate to severe course (PCDAI $\geq 30$ or $C D A I \geq 220$; $\mathrm{p}=0.027$ ) of disease significantly more frequent. However, in the adult-onset group disease activity and moderate to severe course measured by CDAI was independent of the NOD2 genotype (Figure 2B).

Pediatric-onset $C D$ is associated with growth delay at diagnosis and during the first year after diagnosis, however only underweight during course of disease is associated with patients carrying NOD2 variant alleles $(\mathrm{p}=0.012$; Figure 3). 
Table 1 Characteristics of 202 patients with pediatric- and adult-onset Crohn's disease according to the Montreal classification [22] regarding NOD2 genotype at latest follow-up

\begin{tabular}{|c|c|c|c|c|c|c|}
\hline \multirow{3}{*}{$\begin{array}{r}\text { Patients } \\
\mathrm{n}=202\end{array}$} & \multicolumn{3}{|c|}{ Pediatric-onset CD ( $\leq 18$ y) } & \multicolumn{3}{|c|}{ Adult-onset CD (>18 y) } \\
\hline & \multicolumn{3}{|c|}{$n=85$} & \multicolumn{3}{|c|}{$n=117$} \\
\hline & Main variants & Wild-type & p-value & Main variants & Wild-type & p-value \\
\hline Number: $\mathrm{n}(\%)$ & 37 (44\%) & $48(56 \%)$ & & $47(40 \%)$ & $70(60 \%)$ & 0.67 \\
\hline 1 variant allele & $23(27 \%)$ & & & $37(32 \%)$ & & \\
\hline 2 variant alleles & $14(16 \%)$ & & & $10(9 \%)$ & & 0.12 \\
\hline homozygous variant alleles & $7(8 \%)$ & & & $3(3 \%)$ & & 0.099 \\
\hline Gender: female/male (\%) & $57 / 43$ & $38 / 62$ & 0.08 & $51 / 49$ & $59 / 41$ & 0.39 \\
\hline \multicolumn{7}{|l|}{ Age: year } \\
\hline mean/SD & $26 / 13.0$ & $22.7 / 12.6$ & 0.26 & $47.3 / 14.3$ & $46.5 / 13.4$ & 0.67 \\
\hline median/interquartile range & $22 / 17-35$ & $20 / 15-26$ & 0.18 & $46 / 36-62$ & $45 / 36-54$ & 0.78 \\
\hline average disease duration/SD & $12.8 / 11.2$ & $11.3 / 10.5$ & 0.63 & $16.2 / 11.3$ & $16.5 / 10.3$ & 0.89 \\
\hline \multicolumn{7}{|l|}{ Age at diagnosis: year } \\
\hline mean/SD & $13.9 / 3.3$ & $12.1 / 4.9$ & 0.07 & $31.5 / 10.0$ & $30.5 / 10.6$ & 0.49 \\
\hline median/interquartile range & $15.0 / 12-17$ & $13.0 / 9-17$ & 0.18 & $27 / 23-33$ & $29 / 24-38$ & 0.32 \\
\hline \multicolumn{7}{|l|}{ Localization: n (\%) } \\
\hline L1 ileal & $9(24 \%)$ & $12(25 \%)$ & 1.0 & $18(38 \%)$ & $14(21 \%)$ & 0.058 \\
\hline L2 colonic & $3(8 \%)$ & $13(27 \%)$ & $0.048^{*}$ & $4(8 \%)$ & $11(16 \%)$ & 0.27 \\
\hline L3 ileocolonic & $26(68 \%)$ & $23(48 \%)$ & $0.048^{*}$ & $25(53 \%)$ & $41(61 \%)$ & 0.35 \\
\hline L4 isolated upper disease & $0(0 \%)$ & $0(0 \%)$ & 1.0 & $0(0 \%)$ & $1(1 \%)$ & 1.0 \\
\hline Upper Gl involvement & $12(32 \%)$ & $16(33 \%)$ & 1.0 & $1(2 \%)$ & $7(10 \%)$ & 0.14 \\
\hline \multicolumn{7}{|l|}{ Behaviour: n (\%) } \\
\hline B1 non-stricturing/penetrating & $16(43 \%)$ & $33(69 \%)$ & $0.026^{*}$ & $16(35 \%)$ & $28(41 \%)$ & 0.53 \\
\hline B2 stricturing & $15(41 \%)$ & $6(13 \%)$ & $0.005^{*}$ & $22(48 \%)$ & $20(29 \%)$ & 0.051 \\
\hline B3 penetrating intern & $6(16 \%)$ & $3(6 \%)$ & 0.16 & $12(26 \%)$ & $11(16 \%)$ & 0.23 \\
\hline B3p penetrating perianal & $7(19 \%)$ & $7(15 \%)$ & 0.76 & $8(17 \%)$ & $19(28 \%)$ & 0.27 \\
\hline
\end{tabular}

* indicates $p$ value $\leq 0.05$ considered to be significant.

Osteoporosis is associated with NOD2 variants, inflammatory course of disease and underweight BMD measured by DEXA was available for 77 pediatricand 91 adult-onset CD patients. Osteoporosis was associated with patients carrying NOD2 variant alleles $(\mathrm{p}=0.033)$ and pediatric-onset of CD $(\mathrm{p}=0.022)$. Multivariate analysis demonstrated a 2.4 times increased risk for osteoporosis in CD patients carrying any NOD2 variant allele $(\mathrm{p}=0.02)$. There was also a suggestive trend for CD patients with NOD2 variant alleles and pediatric-onset towards osteoporosis $(\mathrm{p}=0.12$, Figure 1 ; and by multivariate analysis $\mathrm{p}=0.07$ ) and low BMD (Z-score below $-1 \mathrm{p}=0.10$; Table 2). Especially pediatric onset $C D$ patients with homozygous NOD2 variant alleles are prone to osteoporosis and osteopenia ( $\mathrm{p}=0.037$; Table 2) and lower Z-scores respectively $(\mathrm{p}=0.033)$. We further evaluated the impact of factors independent of the NOD2 genotype influencing BMD. 46 out of 77 patients had a low BMD (Z-score below -1) thereof 23 (30\%) suffered from osteoporosis (Z-score below -2.0) and 23 (30\%) from osteopenia, only 31 (40\%) had normal BMD (Table 2). The average disease duration was around 12 years in the low and normal BMD group. Osteoporosis at diagnosis of CD or in the follow-up was not associated with surgery, complications, extraintestinal manifestation or ileal or ileocolonic involvement (Table 2 and data not shown). However, patients with low BMD Z-scores had a significantly higher PCDAI-score $(\mathrm{p}=0.0092)$ (Figure 4). A multivariate regression model demonstrated that the PCDAI-score is 5.8 points higher in patients with low BMD than normal BMD, whereas this effect is adjusted for steroid dependent or refractory course of disease and underweight $(\mathrm{p}=0.03)$. In addition, underweight at diagnosis $(p=0.045)$ and after 1year of follow up $(p=0.011)$ but not short stature was significant associated with low BMD (Table 2). The need for immunosuppressive 


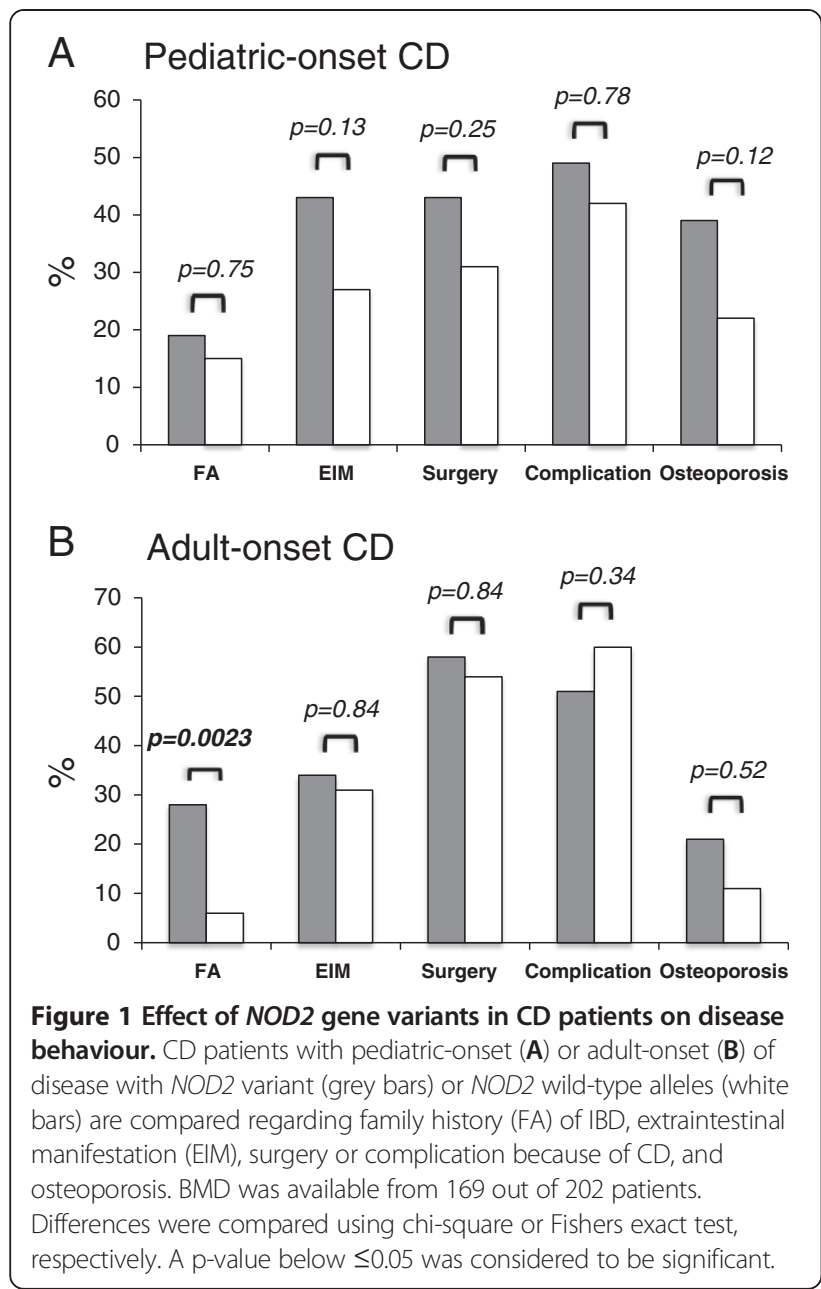

treatment with azathioprin (AZA) or methotrexate (MTX) was associated with low BMD $(\mathrm{p}=0.016)$ and there was a trend to steroid-refractory or dependent course $(\mathrm{p}=0.1)$ or anti-TNF $\alpha$ therapy $(\mathrm{p}=0.09)$. Interestingly, $57 \%$ of the patients with low BMD were steroid- or immunosuppressiva naïve at the time of the first measurement (data not shown).

\section{Influence of NOD2 genotype on therapy regarding age at diagnosis of $C D$}

Comparing therapy and NOD2 genotype revealed divergent trends between pediatric- and adult onset CD. Steroid dependent or refractory course of disease was more frequent in pediatric-onset $\mathrm{CD}$ patients with NOD2 variants $(p=0.048)$ (Figure 5). In addition, the use of long-term immunosuppressive drugs such as antiTNF $\alpha$ agents; azathioprine or methotrexate trend to be higher in patients with NOD2 variant alleles and pediatric onset in contrast to adult-onset CD (Figure 5). This is also confirmed by analyzing the relationship between age of diagnosis, response to therapy and NOD2 genotype. A significant age dependent difference towards therapy was
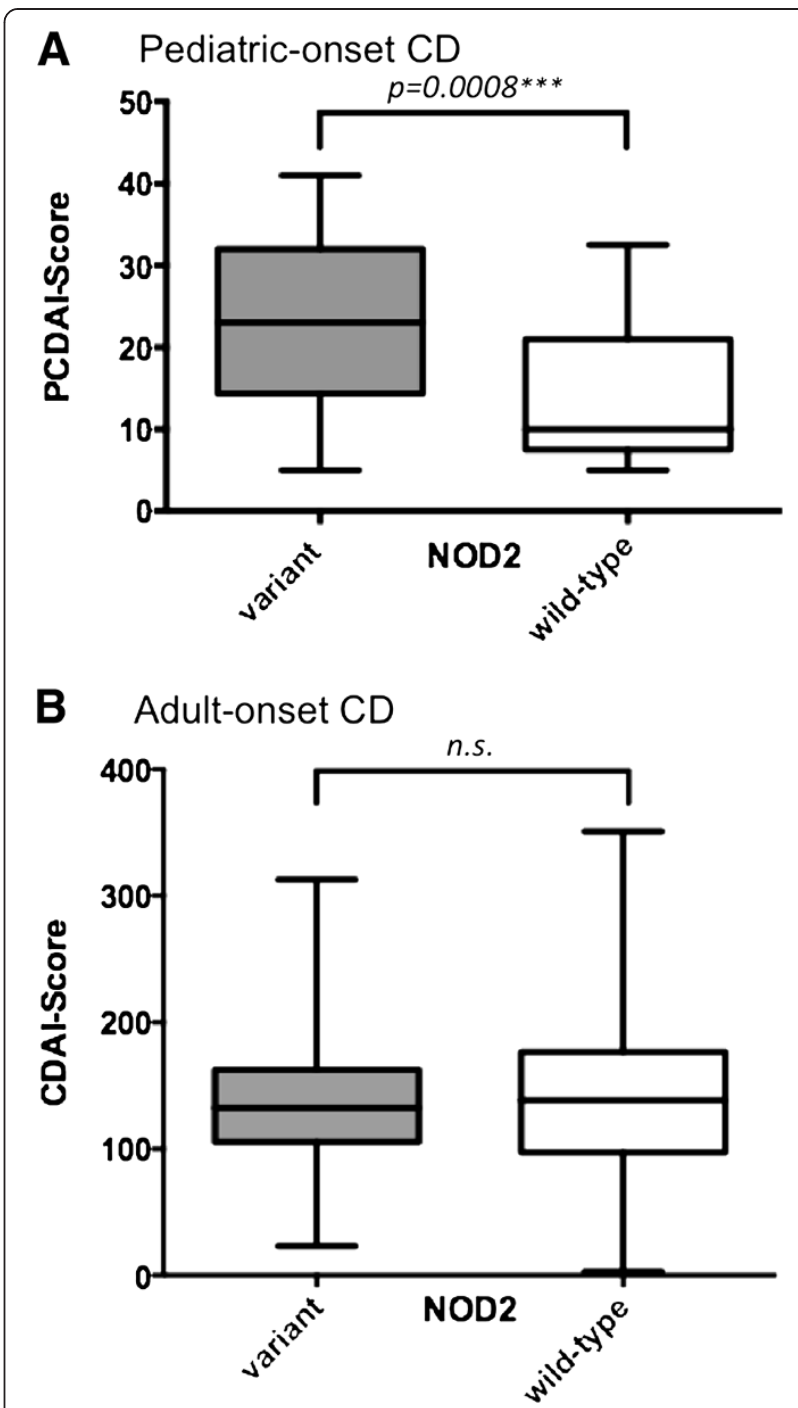

Figure 2 Effect of NOD2 variants on PCDAI and CDAI Score in pediatric- and adult-onset $C D$ patients. $C D$ patients with pediatric-onset (A) and adult-onset (B) are compared regarding NOD2 variant (grey bars) or NOD2 wild-type alleles (white bars) and their average PCDAI or CDAI Score. The median (lines within the boxes), the interquartile range (boxes) and the whole range are given. Differences were compared using unpaired, two sided Mann-Whitney test. A p-value below $\leq 0.05$ was considered to be significant.

only found in $\mathrm{CD}$ patients carrying NOD2 variants. CD patients carrying at least one NOD2 variant allele and being young at diagnosis (median age below 20 years) are at risk for steroid dependent or refractory course of disease $(p=0.0022)$, long-term immunosuppressive therapy with AZA or MTX $(p=0.0426)$ and the use of anti-TNF $\alpha$ agents $(\mathrm{p}=0.0004)$ (Additional file 1: Figure S1). In addition, carrying a NOD2 variant allele is associated with a 3.1 increased risk for steroid dependent or refractory course of disease $(\mathrm{p}=0.02)$. 


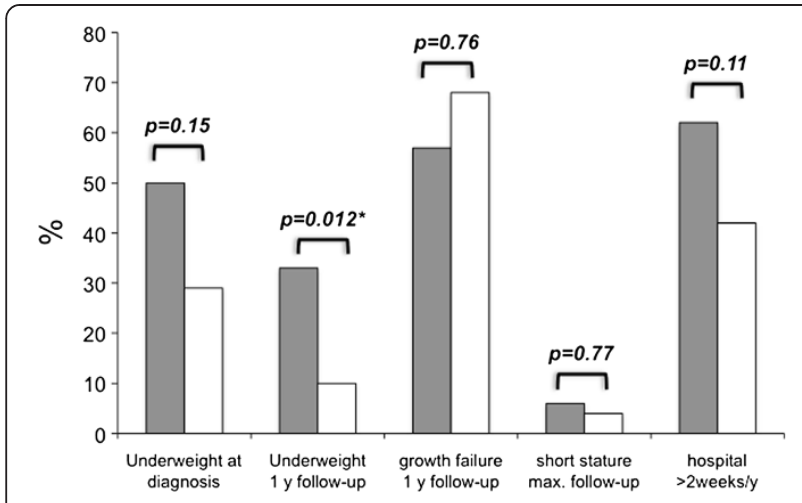

Figure 3 Effect of NOD2 variants on underweight, growth failure, short stature and hospitalization in pediatric-onset $C D$ patients. Pediatric-onset CD patients with NOD2 variant (grey bars) or NOD2 wild-type alleles (white bars) were compared regarding underweight at diagnosis and during follow up, growth failure, short stature and hospitalization for more than two weeks per year. BMI at diagnosis and growth delay at one-year follow-up was available from 51 out of 85 patients (39\% NOD2 variant allele carriers). Differences were compared using chi-square or Fishers exact test, respectively. A p-value below $\leq 0.05$ was considered to be significant.

\section{Discussion}

Evaluation of disease behaviour by genotype is complex and may change throughout the course of disease. Dissecting pediatric- and adult-onset CD represents a model to identify further associations between NOD2 gene variants and phenotypes and may help in selecting individual treatment regimes for patients with $\mathrm{CD}$. We here demonstrate greater influence of NOD2 variants in pediatric-onset $\mathrm{CD}$ patients towards higher disease activity, risk for underweight, osteoporosis and more intensive immunosuppressive therapy. This is the first study identifying an influence of NOD2 variant alleles in patients with $\mathrm{CD}$ on osteoporosis and an age of onset dependent risk for steroid dependent or refractory course, long-term use of immunosuppressive drugs.

In this retrospective single center study in a tertiary center we report a high prevalence of $\mathrm{CD}$ patients carrying at least one NOD2 variant allele with $44 \%$ in the pediatricand $42 \%$ in adult-onset CD similar to other European pediatric multicenter cohort studies with a prevalence of 35 up to $45.6 \%$ [12,23,24]. In addition, the presence of two NOD2 mutant alleles was found more frequent in the pediatric-onset $\mathrm{CD}$ patients as reported by others [23,25] and may predict complicated disease [26].

Growth retardation is a frequent specific feature of pediatric-onset $C D$ [27], which manifest as poor weight gain or short stature and may be the first presenting symptom. We observed a trend in $\mathrm{CD}$ patients with NOD2 variants towards underweight at diagnosis in our pediatric-onset cohort analogous to other studies [28-30] describing lower weight and a tendency to growth retardation more or less pronounced in their pediatric
Table 2 Characteristics of 77 Crohn patients with pediatric-onset according to the Montreal classification regarding BMD adjusted for age

\begin{tabular}{|c|c|c|c|}
\hline Pediatric-onset CD patients & Low BMD & Normal BMD & \\
\hline & Z-score $<-1$ & Z-score $\geq-1$ & p-value \\
\hline $\mathrm{n}=77$ & $n=46$ & $\mathrm{n}=31$ & \\
\hline$\%$ & $60 \%$ & $40 \%$ & \\
\hline NOD2 variant $\mathrm{n}(\%)$ & $24(52 \%)$ & $10(32 \%)$ & $p=0.10$ \\
\hline One variant allele & 17 & 5 & $p=0.061$ \\
\hline Two variant alleles & 7 & 5 & $p=0.75$ \\
\hline Homozygous variant alleles & 7 & 0 & $p=0.037^{*}$ \\
\hline Gender: female/male in \% & $45 / 55$ & $48 / 52$ & $p=0.89$ \\
\hline \multicolumn{4}{|l|}{ Age: $y$} \\
\hline mean/SD & $25 / 12.8$ & $24 / 13.9$ & $p=0.62$ \\
\hline median/interquartile range & $21 / 17-30$ & $19 / 15-29$ & $p=0.29$ \\
\hline average disease duration/SD & $12.2 / 11.6$ & $11.6 / 10.8$ & $p=0.84$ \\
\hline \multicolumn{4}{|l|}{ Age at diagnosis: $y$} \\
\hline mean/SD & $13 / 3.6$ & $12 / 5.1$ & $p=0.33$ \\
\hline median/interquartile range & $14 / 11-16$ & $13 / 10-17$ & $p=0.69$ \\
\hline \multicolumn{4}{|l|}{ Localization: n (\%) } \\
\hline L1 ileal & $8(17 \%)$ & $9(29 \%)$ & $p=0.27$ \\
\hline L2 colonic & $8(17 \%)$ & $6(19 \%)$ & $p=1.0$ \\
\hline L3 ileocolonic & $30(65 \%)$ & $16(52 \%)$ & $p=0.25$ \\
\hline L4 isolated upper disease & $0(0 \%)$ & $0(0 \%)$ & $p=1.0$ \\
\hline Upper GI involvement & $15(33 \%)$ & $13(42 \%)$ & $p=0.47$ \\
\hline
\end{tabular}

Behaviour: $\mathbf{n}$ (\%)

B1 non-stricturing/penetrating

B2 stricturing

$27(59 \%) \quad 20(65 \%) \quad p=0.64$

B3 penetrating intern

$11(23 \%) \quad 6(19 \%) \quad p=0.78$

B3p penetrating perianal

$6(13 \%) \quad 3(10 \%)$

$\mathrm{p}=0.73$

Underweight at diagnosis

$9(19 \%)$

$3(10 \%)$

$\mathrm{p}=0.34$

Underweight in 1y follow-up

14/27 (52\%) 5/22 (32\%)

$\mathrm{p}=0.0453^{*}$

Short stature

$14(30 \%)$

$2(6 \%)$

$p=0.0113^{*}$

Treatment: $\mathbf{n}$ (\%)

Steroid dependent/refractory

$5(11 \%)$

$1(3 \%)$

$\mathrm{p}=0.39$

Azathioprine / MTX

$23(50 \%) \quad 9(29 \%) \quad \mathrm{p}=0.10$

Anti-TNFa-antibody

$35(76 \%) \quad 15(48 \%)$

$\mathrm{p}=0.016^{*}$

${ }^{*}$ indicates $p$ value $\leq 0.05$ considered to be significant.

cohorts. In addition, we found also significant ongoing underweight in the course of disease in patients with NOD2 variant alleles. Growth failure seems to be associated with disease severity [29] and high levels of TNFo in pediatric IBD [31]. Indeed, the average disease activity in pediatric-onset $\mathrm{CD}$ patients carrying at least one NOD2 variant allele was significantly higher and associated with chronic active or moderate to severe clinical course of disease. However, other studies did not found an influence of NOD2 variants towards higher disease 


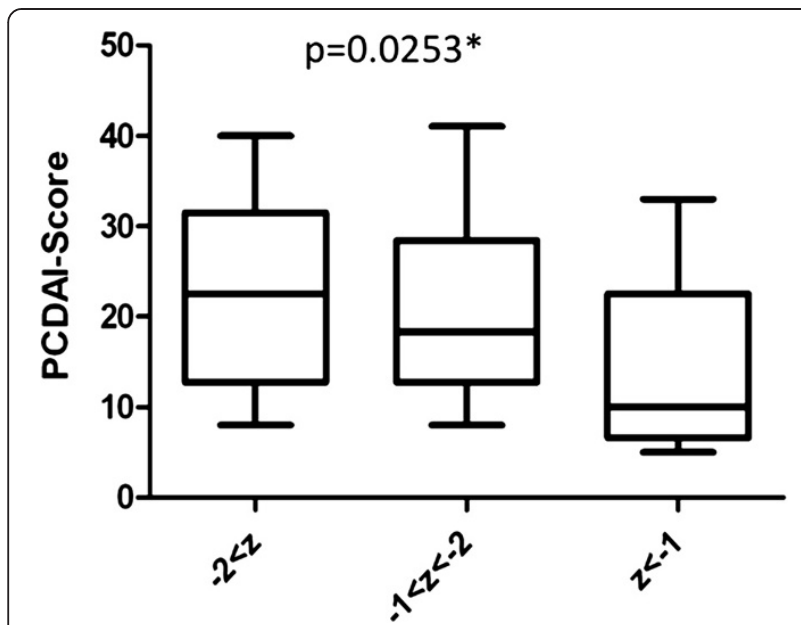

Figure 4 Effect of PCDAI on BMD. CD patients with pediatriconset are compared regarding their PCDAl and BMD z-value. The median (lines within the boxes), the interquartile range (boxes) and the whole range are given. Differences were compared using Kruskal-Wallis test. The medians varies significant $\left(^{*}\right)$, p-value is 0.0253 using Gaussian approximation.

activity $[25,29]$. This is explicable by the fact, that in most instances disease activity was educed from hospitalization, need for immunosuppressive drugs, surgery or occurrence of complications but not by PCDAI at onset and during course of disease [25,28,29,32]. The PCDAI-score was only used by one other pediatric study with $65 \mathrm{CD}$ patients, which described an influence of NOD2 variants towards a higher PCDAI [30]. Besides actual history and laboratory values (e.g. haematocrit, erythrocyte sedimentation rate and albumin) the PCDAI score includes also weight loss, growth delay, perirectal disease and extraintestinal manifestation. Therefore a high PCDAI score in our pediatric-onset $\mathrm{CD}$ patients with NOD2 variants reflects a more severe disease behaviour indicated by underweight, stricturing and penetrating behaviour. Moreover, an aggressive disease manifestation in pediatric-onset $\mathrm{CD}$ patients with $N O D 2$ variants leading to complications and surgical resection is described by Lacher et al. [12] and Kugathasan et al. [32,33].

The prevalence of osteoporosis ranges from 7 to $30 \%$ and osteopenia from 22 to $70 \%$ in pediatric IBD studies $[13,34,35]$. In fact, $30 \%$ of our patients with pediatric-onset CD have osteoporosis and $31 \%$ osteopenia, some even before corticosteroid treatment confirming data from Walther [13] and Harpavat et al. [36]. Possible risk factors for pathologic bone density in $\mathrm{CD}$ are disease activity, lifetime steroid dosage over $10 \mathrm{~g}$, CD, multiple bowel resections, young age and low body mass index according to a large epidemiological study [37]. We also observed low BMI and a higher disease activity associated with reduced bone density in pediatric-onset CD. Similar Paganelli et al. described an inversely correlation of

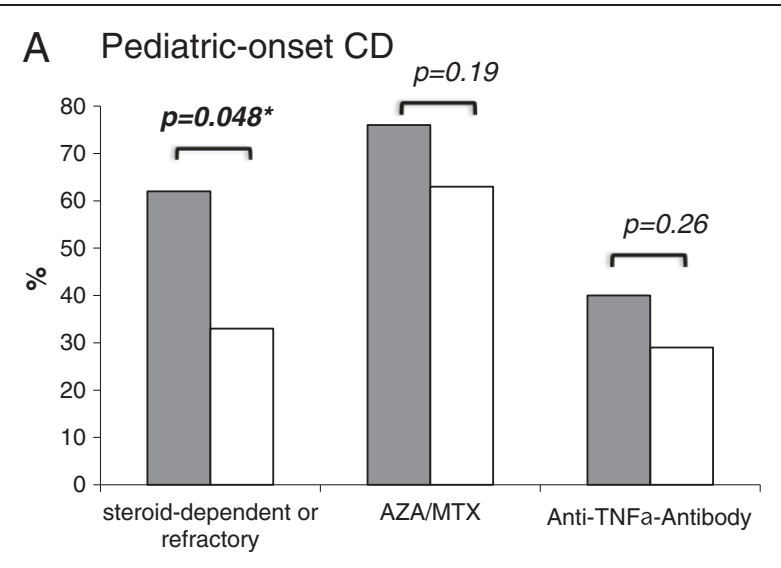

\section{B Adult-onset CD}

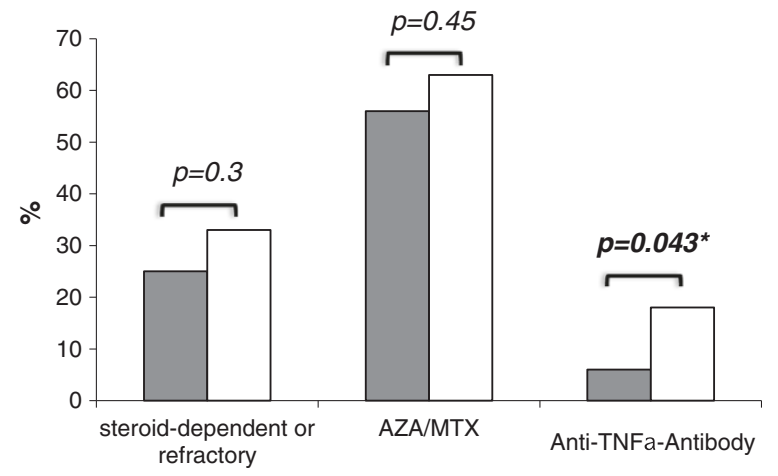

Figure 5 Effect of NOD2 variant alleles on treatment in pediatric- and adult-onset $C D$. CD patients with pediatric-onset (A) or adult-onset (B) of disease with NOD2 variant (grey bars) or NOD2 wild-type alleles (white bars) are compared regarding steroiddependent or refractory course of disease, need for long-term immunosuppressive therapy with azathioprine (AZA) and methotrexate (MTX) or anti-TNFa antibody. Differences were compared using chi-square or Fishers exact test, respectively. A p-value below $\leq 0.05$ was considered to be significant.

volumetric BMD with disease activity measured by PCDAI and IL-6 [38]. Other risk factors such as ileal localization and bowel resection did not play a role in our pediatriconset cohort. Lifetime corticosteroid treatment in children with IBD seems to be less important as previously believed, as children with a chronic non-inflammatory type of disease such as steroid sensitive nephritic syndrome (SSNS) receiving glucocorticoids chronically do have a better bone mineral density than children with $\mathrm{CD}$ [39]. Certainly osteoporosis was associated with a steroid refractory or dependent course of disease in the pediatric-onset group. In fact the glucocorticoid dosage in the treatment of pediatric CD may be not as high as in previous older studies; children with $\mathrm{CD}$ often receive enteral nutrition therapy and there are more patients with steroid dependent or refractory course of disease in the low BMD group actually switched earlier to a long-term immunosuppressive therapy including azathioprine, methotrexate and 
anti-TNF- $\alpha$ antibody treatment. Thus the inflammatory state in $\mathrm{CD}$ might basically contribute to high-turn over bone mineral loss. It is also known that NOD2 variant alleles induce an inflammatory response e.g. via NF-KB activation [40]. Thus some cytokines with osteolytic activity such as TNF $\alpha$, IL-1 $\beta$, IL-6, IFN $\gamma$, RANK, RANKL and osteoprotegrin may also be elevated. IL-1 $\beta$ for example stimulates osteoclast activity and enhances mineral bone loss. In fact carriers of $I L 1 \beta-511 b$ allele have significant lower BMD [41]. Interestingly, CD patients carrying NOD2 variant alleles have an increased risk towards osteoporosis. This is more pronounced in pediatric-onset $\mathrm{CD}$, especially in patients carrying homozygous NOD2 variant alleles. However, we could not prove our hypothesis that osteoporosis is significantly more frequent in pediatric-onset CD patients carrying any NOD2 variant allele may be due to a lack of power. Taken together, these data support screening of osteoporosis in pediatric onset $\mathrm{CD}$, especially in children with $N O D 2$ variants, low BMI and high PCDAI.

We recently reported that $\mathrm{CD}$ patients with NOD2 carrier status were more refractory for steroid treatment but responded well to immunomodulators such as AZA/ 6-MP [42]. This was in contrast to another study that could not find an association between NOD2 carrier status or age of onset of disease and response to steroids [43]. We speculated that treatment response might vary analyzing pediatric and adult $\mathrm{CD}$ patient study cohorts regarding NOD2 genotype. To corroborate this hypothesis we now included a pediatric $\mathrm{CD}$ cohort and reclassified all $\mathrm{CD}$ patients in our adult cohort according to the onset of disease in pediatric and adult onset. Using this approach we found a significant association for steroid failure in $C D$ patients with NOD2 variant alleles and younger age of onset in contrast to Weiss et al. [43]. In addition, the need for long-term immunosuppressive therapy and second line therapy with anti-TNF-alpha agents was also only associated with younger age of onset and the presence of any NOD2 variant allele. Although a positive response to the anti-TNF $\alpha$ agent infliximab was found to be associated with a higher systemic inflammation [44], the NOD2 genotype was not predictive of treatment outcome in their cohort [45]. The diverging results regarding our study cohort might be due to the subgroup analysis of pediatric-onset $C D$. The fact that we did not investigate treatment outcome is another disadvantage of this study and its retrospective approach.

\section{Conclusion}

In summary, dissecting a large unselected group of patients with long-term $\mathrm{CD}$ duration in pediatric- and adult-onset of disease revealed a more pronounced difference between NOD2 genotype and phenotype in the pediatric-onset group. There was an association of NOD2 gene variants towards a more affected phenotype with underweight, higher disease activity, steroid failure and the need for long-term and intensive immunosuppressive therapy. Thus pediatric-onset $\mathrm{CD}$ and NOD2 variant alleles are risk factors frequently associated with chronic and moderate to severe course of disease. In addition, the prevalence of low bone mineral density is considerably high in pediatric Crohn patients with high average PCDAI, low BMI, steroid failure and NOD2 variant alleles.

The major goal in the treatment of $\mathrm{CD}$ is to change the fatal course of chronic active disease especially in children and adolescents in order to improve growth and prevent osteoporosis. Until now testing for NOD2 variants is not part of the diagnostic work-up in pediatric-onset $\mathrm{CD}$. We conclude from our study, that genetic testing for NOD2 variant alleles is useful in pediatric-onset $\mathrm{CD}$ as it may identify patients who are at risk for a more affected phenotype. Given the beneficial effect of TNF- $\alpha$ blockade on bone formation and on CD activity this may be an alternative early treatment strategy in children $[5,46]$.

\section{Additional file}

Additional file 1: Figure S1. Effect of age of onset at diagnosis of CD and NOD2 variants on treatment. Th age of onset of CD in 202 patient with NOD2 variant (grey bars) or wild-type (white bars) alleles is compared regarding steroid-dependent or refractory course of disease (A), need for long-term immunosuppressive therapy with azathioprine (AZA) and methotrexate (MTX) (B) or anti-TNFa agents (C). Differences were compared using Kruskal-Wallis test. A p-value below $\leq 0.05$ was considered to be significant and indicated with an asterix.

\section{Abbreviations}

6-MP: 6-Mercaptopurine; AZA: Azathioprine; BMD: Bone mineral density; CARD 15: Caspase-activation recruitment domains 15; CD: Crohn's disease; CDAl: Crohn's disease activity index; IBD: Inflammatory bowel disease; SNP: Single nucleotide polymorphism; NOD2: Nucleotide oligomeric domain 2; PCDAl: Pediatric Crohn's activity index; SNP: Single-nucleotide polymorphism; TNF: Tumor necrosis factor; UC: Ulcerative colitis; WT: Wild-type.

\section{Competing interest}

There is no potential conflict of interest of any author relevant to the manuscript.

\section{Authors' contributions}

CP conceived of the study, participated in the coordination and collection of data, performed the statistical analysis and drafted the manuscript. GL carried out the molecular genetic studies. VP, JHN and JK carried out the coordination and data collection. BM performed the statistical analysis. GvB and KMD participated in the design and coordination of the study. All authors read and approved the final manuscript.

\section{Author details}

'Department of Pediatrics and Adolescent Medicine, University Medical Center Ulm, Eythstr. 24, UIm 89075, Germany. ${ }^{2}$ Internal Medicine, University Medical Center Ulm, Albert Einstein Allee 24, Ulm 89081, Germany. ${ }^{3}$ Medical department of the Academic Clinic Sigmaringen, Hohenzollernstr. 40, Sigmaringen 72488, Germany. ${ }^{4}$ Institute of Epidemiology and Medical Biometry, Ulm University, Ulm, Germany.

Received: 7 August 2012 Accepted: 26 April 2013

Published: 2 May 2013 


\section{References}

1. Orholm M, Binder V, Sorensen TI, Rasmussen LP, Kyvik KO: Concordance of inflammatory bowel disease among Danish twins. Results of a nationwide study. Scand J Gastroenterol 2000, 35(10):1075-1081.

2. Biank V, Broeckel U, Kugathasan S: Pediatric inflammatory bowel disease: clinical and molecular genetics. Inflamm Bowel Dis 2007, 13(11):1430-1438.

3. Hampe J, Cuthbert A, Croucher PJ, Mirza MM, Mascheretti S, Fisher S, Frenzel H, King K, Hasselmeyer A, MacPherson AJ, et al: Association between insertion mutation in NOD2 gene and Crohn's disease in German and British populations. Lancet 2001, 357(9272):1925-1928.

4. Hampe J, Grebe J, Nikolaus S, Solberg C, Croucher PJ, Mascheretti S, Jahnsen J, Moum B, Klump B, Krawczak M, et al: Association of NOD2 (CARD 15) genotype with clinical course of Crohn's disease: a cohort study. Lancet 2002, 359(9318):1661-1665.

5. Hugot JP, Chamaillard M, Zouali H, Lesage S, Cezard JP, Belaiche J, Almer S, Tysk C, O'Morain CA, Gassull M, et al: Association of NOD2 leucine-rich repeat variants with susceptibility to Crohn's disease. Nature 2001, 411(6837):599-603

6. Inohara N, Ogura Y, Fontalba A, Gutierrez O, Pons F, Crespo J, Fukase K, Inamura S, Kusumoto S, Hashimoto M, et al: Host recognition of bacterial muramyl dipeptide mediated through NOD2. Implications for Crohn's disease. J Biol Chem 2003, 278(8):5509-5512.

7. Kobayashi KS, Chamaillard M, Ogura Y, Henegariu O, Inohara N, Nunez G, Flavell RA: Nod2-dependent regulation of innate and adaptive immunity in the intestinal tract. Science 2005, 307(5710):731-734

8. Voss E, Wehkamp J, Wehkamp K, Stange EF, Schroder JM, Harder J: NOD2/ CARD15 mediates induction of the antimicrobial peptide human beta-defensin-2. J Biol Chem 2006, 281(4):2005-2011.

9. Cosnes J, Beaugerie L, Carbonnel F, Gendre JP: Smoking cessation and the course of Crohn's disease: an intervention study. Gastroenterology 2001, 120(5):1093-1099.

10. Freeman HJ: Long-term prognosis of early-onset Crohn's disease diagnosed in childhood or adolescence. Can J Gastroenterol 2004, 18(11):661-665.

11. Russell RK, Drummond HE, Nimmo EE, Anderson N, Smith L, Wilson DC, Gillett PM, McGrogan P, Hassan K, Weaver LT, et al: Genotype-phenotype analysis in childhood-onset Crohn's disease: NOD2/CARD15 variants consistently predict phenotypic characteristics of severe disease. Inflamm Bowel Dis 2005, 11(11):955-964.

12. Lacher M, Helmbrecht J, Schroepf S, Koletzko S, Ballauff A, Classen M, Uhlig H, Hubertus J, Hartl D, Lohse P, et al: NOD2 mutations predict the risk for surgery in pediatric-onset Crohn's disease. J Pediatr Surg 2010, 45(8):1591-1597.

13. Walther F, Fusch C, Radke M, Beckert S, Findeisen A: Osteoporosis in pediatric patients suffering from chronic inflammatory bowel disease with and without steroid treatment. J Pediatr Gastroenterol Nutr 2006, 43(1):42-51.

14. Tilg H, Moschen AR, Kaser A, Pines A, Dotan I: Gut, inflammation and osteoporosis: basic and clinical concepts. Gut 2008, 57(5):684-694.

15. Cravo M, Guerreiro CS, dos Santos PM, Brito M, Ferreira P, Fidalgo C, Tavares L, Pereira AD: Risk factors for metabolic bone disease in Crohn's disease patients. Inflamm Bowel Dis 2010, 16(12):2117-2124.

16. Turk N, Cukovic-Cavka S, Korsic M, Turk Z, Vucelic B: Proinflammatory cytokines and receptor activator of nuclear factor kappaB-ligand /osteoprotegerin associated with bone deterioration in patients with Crohn's disease. Eur J Gastroenterol Hepatol 2009, 21(2):159-166.

17. Agrawal M, Arora S, Li J, Rahmani R, Sun L, Steinlauf AF, Mechanick Jl, Zaidi $\mathrm{M}$ : Bone, inflammation, and inflammatory bowel disease. Curr Osteoporos Rep 2011, 9(4):251-257.

18. Hyams J, Markowitz J, Otley A, Rosh J, Mack D, Bousvaros A, Kugathasan S, Pfefferkorn M, Tolia V, Evans J, et al: Evaluation of the pediatric crohn disease activity index: a prospective multicenter experience. J Pediatr Gastroenterol Nutr 2005, 41(4):416-421.

19. Faulkner RA, Bailey DA, Drinkwater DT, McKay HA, Arnold C, Wilkinson AA: Bone densitometry in Canadian children 8-17 years of Age. Calcif Tissue Int 1996, 59(5):344-351.

20. Abitbol V, Roux C, Chaussade S, Guillemant S, Kolta S, Dougados M, Couturier D, Amor B: Metabolic bone assessment in patients with inflammatory bowel disease. Gastroenterology 1995, 108(2):417-422.

21. Hoffmann JC, Preiss JC, Autschbach F, Buhr HJ, Hauser W, Herrlinger K, Hohne W, Koletzko S, Krieglstein CF, Kruis W, et al: [Clinical practice guideline on diagnosis and treatment of Crohn's disease]. $Z$ Gastroenterol 2008, 46(9):1094-1146.
22. Silverberg MS, Satsangi J, Ahmad T, Arnott ID, Bernstein CN, Brant SR, Caprilli R, Colombel JF, Gasche C, Geboes K, et al: Toward an integrated clinical, molecular and serological classification of inflammatory bowel disease: Report of a Working Party of the 2005 Montreal World Congress of Gastroenterology. Can J Gastroenterol 2005, 19(Suppl A):5-36.

23. de Ridder L, Weersma RK, Dijkstra G, van der Steege G, Benninga MA, Nolte IM, Taminiau JA, Hommes DW, Stokkers PC: Genetic susceptibility has a more important role in pediatric-onset Crohn's disease than in adult-onset Crohn's disease. Inflamm Bowel Dis 2007, 13(9):1083-1092.

24. Sun L, Roesler J, Rosen-Wolff A, Winkler U, Koch R, Thurigen A, Henker J: CARD15 genotype and phenotype analysis in 55 pediatric patients with Crohn disease from Saxony, Germany. J Pediatr Gastroenterol Nutr 2003, 37(4):492-497.

25. Lesage S, Zouali H, Cezard JP, Colombel JF, Belaiche J, Almer S, Tysk C, O'Morain C, Gassull M, Binder V, et al: CARD15/NOD2 mutational analysis and genotype-phenotype correlation in 612 patients with inflammatory bowel disease. Am J Hum Genet 2002, 70(4):845-857.

26. Adler J, Rangwalla SC, Dwamena BA, Higgins PD: The prognostic power of the NOD2 genotype for complicated Crohn's disease: a meta-analysis. Am J Gastroenterol 2011, 106(4):699-712.

27. Puntis J, McNeish AS, Allan RN: Long term prognosis of Crohn's disease with onset in childhood and adolescence. Gut 1984, 25(4):329-336.

28. Tomer G, Ceballos C, Concepcion E, Benkov KJ: NOD2/CARD15 variants are associated with lower weight at diagnosis in children with Crohn's disease. Am J Gastroenterol 2003, 98(11):2479-2484.

29. Wine E, Reif SS, Leshinsky-Silver E, Weiss B, Shaoul RR, Shamir R, Wasserman D, Lerner A, Boaz M, Levine A: Pediatric Crohn's disease and growth retardation: the role of genotype, phenotype, and disease severity. Pediatrics 2004, 114(5):1281-1286.

30. Roesler J, Thurigen A, Sun L, Koch R, Winkler U, Laass MW, Gahr M, RosenWolff A, Henker J: Influence of CARD15 mutations on disease activity and response to therapy in 65 pediatric Crohn patients from Saxony, Germany. J Pediatr Gastroenterol Nutr 2005, 41(1):27-32.

31. Murch SH, Lamkin VA, Savage MO, Walker-Smith JA, MacDonald TT: Serum concentrations of tumour necrosis factor alpha in childhood chronic inflammatory bowel disease. Gut 1991, 32(8):913-917.

32. Annese V, Lombardi G, Perri F, D'Inca R, Ardizzone S, Riegler G, Giaccari S, Vecchi M, Castiglione F, Gionchetti P, et al: Variants of CARD15 are associated with an aggressive clinical course of Crohn's disease-an IG-IBD study. Am J Gastroenterol 2005, 100(1):84-92.

33. Kugathasan S, Collins N, Maresso K, Hoffmann RG, Stephens M, Werlin SL, Rudolph C, Broeckel U: CARD15 gene mutations and risk for early surgery in pediatric-onset Crohn's disease. Clin Gastroenterol Hepatol 2004, 2(11):1003-1009

34. Issenman RM, Atkinson SA, Radoja C, Fraher L: Longitudinal assessment of growth, mineral metabolism, and bone mass in pediatric Crohn's disease. J Pediatr Gastroenterol Nutr 1993, 17(4):401-406.

35. Herzog D, Bishop N, Glorieux F, Seidman EG: Interpretation of bone mineral density values in pediatric Crohn's disease. Inflamm Bowel Dis 1998, 4(4):261-267.

36. Harpavat M, Greenspan SL, O'Brien C, Chang CC, Bowen A, Keljo DJ: Altered bone mass in children at diagnosis of Crohn disease: a pilot study. J Pediatr Gastroenterol Nutr 2005, 40(3):295-300.

37. von Tirpitz C, Steder-Neukamm U, Glas K, Sander S, Ring C, Klaus J, Reinshagen M: [Osteoporosis in inflammatory bowel disease - results of a survey among members of the German Crohn's and Ulcerative Colitis Association]. Z Gastroenterol 2003, 41(12):1145-1150.

38. Paganelli M, Albanese C, Borrelli O, Civitelli F, Canitano N, Viola F, Passariello $\mathrm{R}$, Cucchiara S: Inflammation is the main determinant of low bone mineral density in pediatric inflammatory bowel disease. Inflamm Bowel Dis 2007, 13(4):416-423.

39. Leonard MB: Glucocorticoid-induced osteoporosis in children: impact of the underlying disease. Pediatrics 2007, 119(Suppl 2):S166-S174.

40. Inohara N, Nunez G: NODs: intracellular proteins involved in inflammation and apoptosis. Nat Rev Immunol 2003, 3(5):371-382.

41. Nemetz A, Toth M, Garcia-Gonzalez MA, Zagoni T, Feher J, Pena AS, Tulassay $Z$ : Allelic variation at the interleukin 1 beta gene is associated with decreased bone mass in patients with inflammatory bowel diseases. Gut 2001, 49(5):644-649. 
42. Niess JH, Klaus J, Stephani J, Pfluger C, Degenkolb N, Spaniol U, Mayer B, Lahr G, von Boyen GB: NOD2 Polymorphism Predicts Response to Treatment in Crohn's Disease-First Steps to a Personalized Therapy. Dig Dis Sci 2011, 57(4):879-886.

43. Weiss B, Lebowitz O, Fidder HH, Maza I, Levine A, Shaoul R, Reif S, Bujanover $Y$, Karban A: Response to medical treatment in patients with Crohn's disease: the role of NOD2/CRAD15, disease phenotype, and age of diagnosis. Dig Dis Sci 2010, 55(6):1674-1680.

44. Louis E, Vermeire $S$, Rutgeerts P, De Vos M, Van Gossum A, Pescatore P, Fiasse $R$, Pelckmans $P$, Reynaert $H, D^{\prime}$ Haens $G$, et al: A positive response to infliximab in Crohn disease: association with a higher systemic inflammation before treatment but not with -308 TNF gene polymorphism. Scand J Gastroenterol 2002, 37(7):818-824.

45. Vermeire S, Louis E, Rutgeerts P, De Vos M, Van Gossum A, Belaiche J, Pescatore P, Fiasse R, Pelckmans P, Vlietinck R, et al: NOD2/CARD15 does not influence response to infliximab in Crohn's disease. Gastroenterology 2002, 123(1):106-111.

46. Abreu MT, Geller JL, Vasiliauskas EA, Kam LY, Vora P, Martyak LA, Yang H, Hu $B$, Lin YC, Keenan G, et al: Treatment with infliximab is associated with increased markers of bone formation in patients with Crohn's disease. J Clin Gastroenterol 2006, 40(1):55-63.

doi:10.1186/1471-230X-13-77

Cite this article as: Posovszky et al:: Age-of-onset-dependent influence of NOD2 gene variants on disease behaviour and treatment in Crohn's disease. BMC Gastroenterology 2013 13:77.

\section{Submit your next manuscript to BioMed Central and take full advantage of:}

- Convenient online submission

- Thorough peer review

- No space constraints or color figure charges

- Immediate publication on acceptance

- Inclusion in PubMed, CAS, Scopus and Google Scholar

- Research which is freely available for redistribution 